\title{
Stable Maps to Projective Spaces
}

(Dedicated to Professor Masahiro Sugawara on his 60-th birthday)

By

\author{
Kaoru MoRISUGI*
}

\section{§1. Introduction and $\mathbb{R}$ esullts}

Let $\mathbb{H} P^{\infty}$ (resp. $Q^{\infty}$ ) be the infinite dimensional quaternionic projective space (resp. the infinite dimensional quarternionic quasi-projective space). Throughout this note all homology groups have integer coefficients unless explicitly stated. In this paper we study the natural homomorphism from $\{X, Y\}$ to $\operatorname{Hom}\left(H_{*}(X)\right.$, $H_{*}(Y)$ ) when $Y$ is $\mathbb{H} P^{\infty}$ or $Q^{\infty}$, where $\{X, Y\}$ means the based homotopy set of stable maps from $X$ to $Y$. In [6], we determined the image of induced homomorphisms from $\left\{\Sigma^{4 n} \mathbb{H} P^{\infty}, \mathbb{H} P^{\infty}\right\}$ to $\operatorname{Hom}\left(H_{*}\left(\mathbb{H} P^{\infty}\right), H_{*}\left(\mathbb{H} P^{\infty}\right)\right)$.

In case of $Q^{\infty}$, there was a mistake. This mistake was pointed by $\mathrm{H}$. Oshima: the diagram (I) in page 975 in [6] is not commutative. He also proved a corrected theorem by modifying the elements $a(n+s-1) t^{2 n}(n-1)^{s}$ and $f^{\prime}(n, s)$ in [6] so that the diagram (I) commutes. Here we shall give a slightly different statement with him. On the other hand by similar methods in [6], in Theorem 2 we construct various stable maps between projective spaces so that the homology homomorphisms induced by those stable maps give the basis of the image from stable maps to their induced homology homomorphisms. Moreover this method can be extended to cases that the source spaces of stable maps satisfy certain conditions. (See Theorem 1).

I thank very much $\mathrm{H}$. Oshima for giving me a chance of reconsidering about stable maps between projective spaces, and M. Imaoka for valuable conversation with him.

Now we shall give the construction. Let $[X, Y]$ be the based homotopy set and $\{X, Y\}$ the based stable homotopy set. We denote the reduced $K O^{*}$-theory

Communicated by N. Shimada, November 17, 1987.

* Department of Mathematics, Wakayama University, Wakayama 640, Japan. 
of $X$ by $K O^{*}(X)$. Throughout this paper assume that $X$ is a finite complex. However this assumption is not essential. If $X$ is connected, then $K O^{3}(X) \cong$ $[X, S p]$ and $K O^{4}(X) \cong[X, B S p]$, where $S p$ is the infinite dimensional symplectic group and $B S p$ is its classifying space. By James splitting $\theta$ [3] or BeckerSegal splitting $\tau$ [1] [8] (See also [2]), there exist correspondences;

$$
\phi(X): K O^{3}(X) \cong[X, S p] \stackrel{\theta}{\rightarrow}\left[X, \Omega^{\infty} \Sigma^{\infty} Q^{\infty}\right] \cong\left\{X, Q^{\infty}\right\}
$$

and

$$
\psi(X): K O^{4}(X) \cong[X, B S p] \stackrel{\tau}{\rightarrow}\left[X, \Omega^{\infty} \Sigma^{\infty} \boldsymbol{H} P^{\infty}\right] \cong\left\{X, \boldsymbol{H} P^{\infty}\right\}
$$

Let $h:\{X, Y\} \rightarrow \operatorname{Hom}\left(H_{*}(X), H_{*}(Y)\right)$ be the canonical homomorphism obtained by taking the homology induced homomorphisms of stable maps. We denote the composition $h \circ \phi(X)$ (resp. $h \circ \psi(X)$ ) by $\Phi(X)$ (resp. $\Psi(X)$ ). Then we have;

Theorem 1. Let $X$ be a connected finite complex.

1) Assume that $K O^{3}(X)$ is free and that $H^{4 k-1}(X)$ is free for all $k \geqq 1$. Let $\left\{e_{i}\right\}$ be a basis of $K O^{3}(X)$. Then the image of $h:\left\{X, Q^{\infty}\right\} \rightarrow H o m\left(H_{*}(X), H_{*}\left(Q^{\infty}\right)\right)$ is a free abelian group generated by $\left\{\phi(X)\left(e_{i}\right)\right\}_{*}$.

2) Assume that $K O^{4}(X)$ is free and that $H^{4 k}(X)$ is free for all $k \geqq 1$. Let $\left\{w_{i}\right\}$ be a basis of $K O^{4}(X)$. Then the image of $h:\left\{X, \boldsymbol{H} P^{\infty}\right\} \rightarrow H o m\left(H_{*}(X), H_{*}\left(\boldsymbol{H} P^{\infty}\right)\right)$ is a free abelian group generated by $\left\{\psi(X)\left(w_{i}\right)_{*}\right\}$.

Remark. The above theorem can be applied for the complex case after slight modifications. However we leave it to readers. For the cases in $\boldsymbol{H} \boldsymbol{P}^{\infty}$ and $\boldsymbol{C} \boldsymbol{P}^{\infty}$, the above theorem is essentially proved in [4].

Now recall that

$$
K O^{*}\left(\boldsymbol{H} P_{+}^{m-1}\right) \cong K O^{*}\left(S^{0}\right)[x] /\left(x^{m}\right), \quad x \in K O^{4}\left(\boldsymbol{H} P_{+}^{m-1}\right),
$$

and

$$
K O^{*}\left(Q^{m}\right) \cong K O^{*}\left(S^{0}\right)\left\{x_{1}, x_{2}, \cdots, x_{m}\right\}, \quad x_{s} \in K O^{4 s-1}\left(Q^{m}\right)
$$

where $x_{s}$ corresponds to $x^{s-1}$ under the Thom isomorphism: $K O *\left(H P_{+}^{m-1}\right) \cong$ $K O^{*+3}\left(Q^{m}\right)$. Let $\alpha_{i}$ be the standard generator of $K O^{-4 i}\left(S^{0}\right)$. Let $n \geqq 0$ and $m \geqq s \geqq 1$. We denote $\psi\left(\Sigma^{4 n} \boldsymbol{H} P^{m}\right)\left(\alpha_{n+s-1} x^{s}\right)$ by $f(n, s) \in\left\{\Sigma^{4 n} \boldsymbol{H} P^{m}, \boldsymbol{H} P^{\infty}\right.$ and $\phi\left(\Sigma^{4 n} Q^{m}\right)\left(\alpha_{n+s-1} x_{s}\right)$ by $g(n, s) \in\left\{\Sigma^{4 n} Q^{m}, Q^{\infty}\right\}$, where $\alpha_{n+s-1} x^{s} \in K O^{4}\left(\Sigma^{4 n} \boldsymbol{H} P^{m}\right)$ and $\alpha_{n+s-1} x_{s} \in K O^{3}\left(\Sigma^{4 n} Q^{m}\right)$. Similarly we define $t(n, s) \in\left\{\Sigma^{4 n+1} Q^{m}, \boldsymbol{H} P^{\infty}\right\}$ and $u(n, s) \in\left\{\Sigma^{4 n+3} \boldsymbol{H} P^{m}, Q^{\infty}\right\}$ by $\psi\left(\Sigma^{4 n+1} Q^{m}\right)\left(\alpha_{n+s-1} x_{s}\right)$ and $\phi\left(\Sigma^{4 n+3} H P^{m}\right)\left(\alpha_{n+s} x^{s}\right)$ 
respectively. Note that by construction $f(n, s), g(n, s), t(n, s)$ and $u(n, s)$ factor through the obvious stunted spaces; for example $f(n, s)$ comes through the stunted quaternionic projective space $\Sigma^{4 n} \mathbb{H} P_{s}^{m}$.

Also recall that

$$
\begin{aligned}
& H_{*}\left(H P^{\infty}\right) \cong Z\left\{\beta_{1}, \beta_{2}, \cdots\right\}, \operatorname{dim} \beta_{i}=4 i, \\
& H_{*}\left(Q^{\infty}\right) \cong Z\left\{\gamma_{1}, \gamma_{2}, \cdots\right\}, \operatorname{dim} \gamma_{i}=4 i-1,
\end{aligned}
$$

here $\gamma_{i}$ and $\beta_{i}$ are the standard generators.

Theorem 2. Let $n \geqq 0$ and $m \geqq s \geqq 1$. Then,

(1) Image $\Psi\left(\Sigma^{4 n} \mathbb{H} P^{m}\right)$ is a free abelian group generated by $\left\{f(n, s)_{*}\right\}_{m \geqq s \geqq 1}$, and $f(n, s)_{*}$ is determined by

$$
f(n, s)_{*}\left(\beta_{k}\right)=a(n+s-1) \frac{(2 n+2 k) !}{(2 k) !} \frac{(2 s) !}{2} M(k, s) \beta_{n+k} .
$$

(2) Image $\Phi\left(\Sigma^{4 n} Q^{m}\right)$ is a free abelian group generated by $\left\{g(n, s)_{\left.*\}_{m}\right\}_{s} \geqq 1}\right.$, and $g(n, s)_{*}$ is determined by

$$
g(n, s)_{*}\left(r_{k}\right)=a(n+s-1) \frac{(2 n+2 k-1) !}{(2 k-1) !} \cdot(2 s-1) ! M(k, s) r_{n+k} .
$$

(3) Image $\Psi\left(\Sigma^{4 n+1} Q^{m}\right)$ is a free abelian group generated by $\left\{t(n, s)_{*}\right\}_{m \geqq s \geqq 1}$, and $t(n, s)_{*}$ is determined by

$$
t(n, s)_{*}\left(r_{k}\right)=a(n+s-1) \cdot k \cdot \frac{(2 n+2 k) !}{(2 k) !}(2 s-1) ! M(k, s) \beta_{n+k} .
$$

(4) Image $\Phi\left(\Sigma^{4 n+3} \mathbb{H} P^{m}\right)$ is a free abelian group generated by $\left\{u(n, s)_{*}\right\}_{m \geq s \geqq 1}$, and $u(n, s)_{*}$ is determined by

$$
u(n, s)_{*}\left(\beta_{k}\right)=a(n+s) \frac{(2 n+2 k+1) !}{(2 k) !} \cdot(2 s) ! M(k, s) \gamma_{n+k+1} .
$$

Here we used the following convention;

$a(i)=1$ if $i$ is even and $=2$ if $i$ is odd, and integers $M(k, s)$ are defined by

$$
\left(e^{z}+e^{-z}-2\right)^{s}=\sum_{k \geqq 1} \frac{(2 k) !}{(2 s) !} \cdot M(k, s) z^{2 k}
$$

For the properties of the number $M(k, s)$, see [9].

Remark 1. (2) in the above theorem is the corrected one of Theorem 3 of [6]. 
Remark 2. By the naturality of construction those maps $f(n, s), g(n, s)$, $t(n, s)$ and $u(n, s)$ are compatible with respect to $m$.

Remark 3. $f(n, s), g(n, s), t(n, s)$ and $u(n, s)$ give the generators of the free part of $\pi_{4 n+4 s}^{s}\left(\boldsymbol{H} P^{\infty}\right), \pi_{4 n+4 s-1}^{s}\left(Q^{\infty}\right), \pi_{4 n+4 s}^{s}\left(\boldsymbol{H} P^{\infty}\right)$ and $\pi_{4 n+4 s+3}^{s}\left(Q^{\infty}\right)$ respectively. Because $M(s, s)=1$ and those stable maps factors through the obvious stunted spaces.

For convenience we abbreviate $\underset{\leftarrow}{\lim } f(2,1)$ by $f: \Sigma^{8} \boldsymbol{H} P^{\infty} \rightarrow \boldsymbol{H} P^{\infty}, \underset{\leftarrow}{\leftarrow} \lim g(2,1)$ by $g: \Sigma^{8} Q^{\infty} \rightarrow Q^{\infty}$ and $\lim _{\leftarrow} t(0,1)$ by $t: \Sigma Q^{\infty} \rightarrow H P^{\infty}$. For later use, we cite;

Proposition 3. There exist stable maps $f: \Sigma^{8} \boldsymbol{H} P^{\infty} \rightarrow \boldsymbol{H} P^{\infty}, g: \Sigma^{8} Q^{\infty} \rightarrow Q^{\infty}$ and $t: \Sigma Q^{\infty} \rightarrow \mathbb{H} P^{\infty}$ such that

(1) $f_{*}\left(\beta_{k}\right)=\frac{(2 k+4) !}{(2 k) !} \beta_{k+2}$,

(2) $g_{*}\left(r_{k}\right)=\frac{(2 k+3) !}{(2 k-1) !} r_{k+2}$,

(3) $t_{*}\left(\gamma_{k}\right)=k \beta_{k}$.

Our next result is

Theorem 4. The following diagrams are commutative;

(1) $K O^{4}(X) \longrightarrow$ Bott iso. $\longrightarrow K O^{4}\left(\Sigma^{8} X\right)$<smiles>CC(C)C(=O)O</smiles>

$\operatorname{Hom}\left(H_{*}(X), H_{*}\left(\boldsymbol{H} P^{\infty}\right)\right) \stackrel{f_{\sharp}}{\longrightarrow} \operatorname{Hom}\left(H_{*}(X), H_{*+8}\left(\boldsymbol{H} P^{\infty}\right)\right)$,

(2) $K O^{3}(X) \longrightarrow$ Bott iso. $\longrightarrow K O^{3}\left(\Sigma^{8} X\right)$

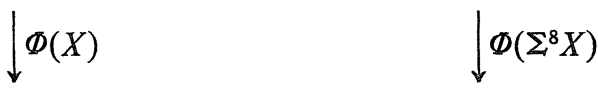

$\operatorname{Hom}\left(H_{*}(X), H_{*}\left(Q^{\infty}\right)\right) \stackrel{g_{\sharp}}{\longrightarrow} \operatorname{Hom}\left(H_{*}(X), H_{*+8}\left(Q^{\infty}\right)\right)$,

(3) $K O^{3}(X) \stackrel{\text { Suspension iso. }}{\longrightarrow} K O^{4}(\Sigma X)$

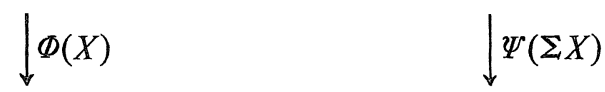

$\operatorname{Hom}\left(H_{*}(X), H_{*}\left(Q^{\infty}\right)\right) \stackrel{t_{*}}{\longrightarrow} \operatorname{Hom}\left(H_{*}(X), H_{*+1}\left(\boldsymbol{H} P^{\infty}\right)\right)$,

where $f_{\sharp}, g_{\sharp}$ and $t_{\sharp}$ are induced by $f_{*}, g_{*}$ and $t_{*}$ respectively. 
Corollary 5. Let $n \geqq 0$ and $s \geqq 1$. Then,

$$
\begin{aligned}
& f(n+2, s)_{*}=f_{*} \circ f(n, s)_{*}, \\
& g(n+2, s)_{*}=g_{*} \circ g(n, s)_{*}, \\
& t_{*} \circ g(n, s)_{*}=t(n, s)_{*}, \\
& t_{*} \circ u(n, s)_{*}=f(n+1, s)_{*}, \\
& f(n, s)_{*} \circ t_{*}=s(t(n, s))_{*} .
\end{aligned}
$$

\section{§2. Proofs}

Let $\eta: \Omega \rightarrow \Omega^{\infty} \Sigma^{\infty} X$ be the natural inclusion. The following Lemma probably well-known. However I have not seen it in the literature.

Lemma 2.1. Let $X$ be a connected $C W$. Let $\left\{a_{i}\right\}$ be a homogeneous basis of $H_{*}(X ; \mathbb{Q})$. Then $H_{*}\left(\Omega^{\infty} \Sigma^{\infty} X ; \mathbb{Q}\right)$ is the free commutative associative algebra generated by $\left\{\eta_{*} a_{i}\right\}$; that is,

$$
H_{*}\left(\Omega^{\infty} \Sigma^{\infty} X ; \mathbb{Q}\right)=\mathbb{P}_{\mathbf{Q}}\left[\eta_{*}\left(a_{\text {even }}\right)\right] \otimes \mathbb{A}_{Q}\left[\eta_{*}\left(a_{\text {odd }}\right)\right] .
$$

Proof. According to [Appendix; 5], $H_{*}\left(\Omega^{\infty} \Sigma^{\infty} X ; \mathbb{Q}\right)$ is a free commutative associative algebra generated by the image of the rational Hurewicz homomorphism $h: \pi_{*}\left(\Omega^{\infty} \Sigma^{\infty} X ; \mathbb{Q}\right) \rightarrow H_{*}\left(\Omega^{\infty} \Sigma^{\infty} X ; \mathbb{Q}\right)$. Now consider the following commutative diagram;

$$
\begin{gathered}
\pi_{*}\left(\Omega^{\infty} \Sigma^{\infty} X ; \mathbb{Q}\right) \stackrel{h}{\longrightarrow} H_{*}\left(\Omega^{\infty} \Sigma^{\infty} X ; \mathbb{Q}\right) \\
\left.\simeq\right|_{\Downarrow} \Sigma \\
\pi_{*}^{r}(X ; \mathbb{Q}) \stackrel{h}{\cong} H_{*}(X ; \mathbb{Q}),
\end{gathered}
$$

where $\sigma$ is the homology suspension and $\Sigma$ is the canonical isomorphism. Thus we see from the above diagram that $\sigma$ induces an isomorphism $H_{*}\left(\Omega^{\infty} \Sigma^{\infty} X ; \mathbb{Q}\right) /$ (decomposables) $\cong H_{*}(X ; \mathbb{Q})$. Since $\sigma\left(h \Sigma^{-1} h^{-1} a_{i}-\eta_{*} a_{i}\right)=0, \eta_{*} a_{i}=h \Sigma^{-1} h^{-1} a_{i}$ mod kernel $\sigma$. Since kernel $\sigma=$ decomposables and $\left\{h \Sigma^{-1} h^{-1} a_{i}\right\}$ are generators as algebra of $H_{*}\left(\Omega^{\infty} \Sigma^{\infty} X ; \mathbb{Q}\right),\left\{\eta_{*} a_{i}\right\}$ gives also generators as algebra of $H^{*}\left(\Omega^{\infty} \Sigma^{\infty} X ; \mathbb{Q}\right)$.

q.e.d.

Lemma 2.2. Let $X$ be a connected finite complex and $h$ be the canonical homomorphism as cited in Theorem 1. Then

(1) Image of $h=$ Image of $\Phi(X)$.

(2) Image of $h=$ Image of $\Psi(X)$. 
Proof. We shall prove (1). The proof of (2) is similar and easier than the proof of (1). So we omit the proof of (2). Let $f \in\left\{X, Q^{\infty}\right\}$. Then it is enough to show that

$$
f_{*}(x)=\left(\sigma \circ \theta_{*} \circ \bar{r}_{*} \circ(\operatorname{adj} f)_{*}\right)(x) \quad \text { for any } \quad x \in H_{*}(X),
$$

where $\sigma: H_{*}\left(\Omega^{\infty} \Sigma^{\infty} Q^{\infty}\right) \rightarrow H_{*}\left(Q^{\infty}\right)$ is the homology suspension, $\bar{r}: \Omega^{\infty} \Sigma^{\infty} Q^{\infty} \rightarrow$ $S p$ is the canonical extension of the reflection map [3] $r: Q^{\infty} \rightarrow S p$ and (adj $f$ ): $X \rightarrow \Omega^{\infty} \Sigma^{\infty} Q^{\infty}$ is the adjoint map of $f$. Since $H_{*}\left(Q^{\infty}\right)$ is torsion free, it is enough to show with rational coefficients. Then from Lemma 2.1 we have

$$
(\operatorname{adj} f)_{*}(x)=\eta_{*}\left(f_{*}(x)\right)+\sum \eta_{*}\left(a_{1}\right) \cdots \eta_{*}\left(a_{n}\right),
$$

where $a_{i} \in H_{*}\left(Q^{\infty} ; \boldsymbol{Q}\right)$ and multiplications are taken in $H_{*}\left(\Omega^{\infty} \Sigma^{\infty} Q^{\infty} ; \boldsymbol{Q}\right)$. Then

$$
\begin{aligned}
\left(\sigma \circ \theta_{*} \circ \bar{r}_{*} \circ(\operatorname{adj} f)_{*}\right)(x) \\
\quad=\sigma \circ \theta_{*} \circ \bar{r}_{*}\left(\eta_{*} f_{*}(x)\right)+\sum \sigma \circ \theta_{*} \circ \bar{r}_{*}\left(\eta_{*}\left(a_{1}\right) \cdots \eta_{*}\left(a_{n}\right)\right) \\
\quad=\sigma\left(\eta_{*} f_{*}(x)\right)+\sum \sigma \circ \theta_{*}\left(r_{*}\left(a_{1}\right) \cdots r_{*}\left(a_{n}\right)\right) \\
\quad=f_{*}(x)+\sum \sigma \circ \theta_{*}\left(r_{*}\left(a_{1}\right) \cdots r_{*}\left(a_{n}\right)\right), \\
\quad=f_{*}(x) .
\end{aligned}
$$

Because, the second and third equation follow from the $\theta \circ \bar{r} \circ \eta=\theta \circ r=\eta[3]$ and that $\sigma \circ \eta_{*}=i d$. and that $\bar{r}_{*}: H_{*}\left(\Omega^{\infty} \Sigma^{\infty} Q^{\infty} ; \boldsymbol{Q}\right) \rightarrow H_{*}(S p ; \boldsymbol{Q})$ is a ring homomorphism. The last equation follows from that $\sigma \circ \theta_{*}$ is equal to $(\operatorname{adj} \theta)_{*}$ under $\Sigma^{\infty}$-isomorphism and that $(\operatorname{adj} \theta)_{*}$ gives the homological decomposition of $H_{*}(S p)[2]$.

q.e.d.

Recall that $H_{*}(B S p)$ is a free abelian group generated by $\beta^{R}$, where $R$ is the usual exponent sequence of non negative integers which are almost all but finite zero. The sequence $\Delta_{i}$ means the sequence of which all entries except the $i$-th are zero and the $i$-th entry is 1 . Let $\left\{P^{R}\right\}, P^{R} \in H^{*}(B S p)$, be the dual basis of $\left\{\beta^{R}\right\}$. Note that $P^{n \Delta_{1}}$ is the usual $n$-th Pontrjagin class $p_{n} \in H^{4 n}(B S p)$. It should be remembered that the Pontrjagin character ph of a symplectic bundle $\xi$ is given by

$$
p h(\xi)=\sum \frac{2}{(2 n) !} P^{\Delta_{n}}(\xi)
$$

We denote the Kronecker pairing by $\langle$,$\rangle . Then,$

Lemma 2.3. Let $X$ be a finite complex.

(1) Let $\xi \in K O^{4}(X)$ and $x \in H_{4 n}(X)$. Then 


$$
\Psi(X)(\xi)(x)=\left\langle x, P^{\Delta_{n}}(\xi)\right\rangle \beta_{n} .
$$

(2) Let $\zeta \in K O^{3}(X)$ and $z \in H_{4 n-1}(X)$. Then,

$$
\Phi(X)(\zeta)(z)=\left\langle\Sigma z, p_{n}(\Sigma \zeta)\right\rangle \gamma_{n}
$$

where $\Sigma z \in H_{4 n}(X)$ and $\Sigma \zeta \in K O^{4}(X)$ are the suspensions of $z$ and $\zeta$.

Proof. (1) is easy. We shall prove (2). We use the same letter for the corresponding map, $\zeta: X \rightarrow S p$, to an element of $\zeta \in K O^{3}(X)$.

Consider the following commutative diagram;

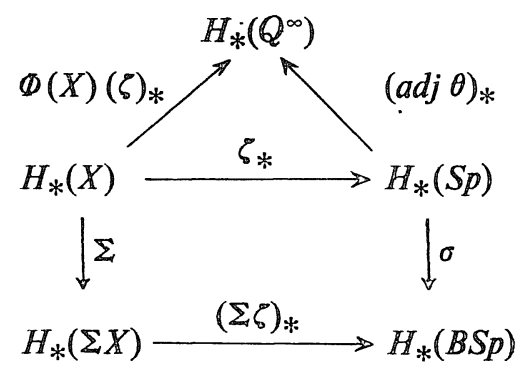

Let $\Phi(X)(\zeta)(z)=\lambda r_{n}$. Then $\zeta_{*}(z)=\lambda r_{n}+$ decomposables in $H_{*}(S p)$. Thus, $(\Sigma \zeta)_{*}(\Sigma z)=\sigma\left(\zeta_{*} z\right)=\lambda \sigma\left(r_{n}\right)$. Since $\left\langle\sigma\left(\gamma_{n}\right), p_{n}\right\rangle=1, \lambda=\left\langle(\Sigma \zeta)_{*}(\Sigma z), p_{n}\right\rangle=$ $\left\langle\Sigma z, p_{n}(\Sigma \zeta)\right\rangle$.

q.e.d.

Proof of Theorem 1. Take an element $\xi \in K O^{3}(X)$. Let $\delta \in K O^{4}(\Sigma X)$ be the element which corresponds to $\xi$. Let $x \in H_{4 n-1}(X)$ be an arbitrary element. Let $\Phi(X)(\xi)(x)=\lambda \gamma_{n}$. Then by Lemma $2.3 \lambda=\left\langle\Sigma x, p_{n}(\delta)\right\rangle$, where $p_{n}$ is the $n$-th Pontrjagin class of $\delta$. So if $\Phi(X)(\xi)=0$, this implies that for any element $x,\left\langle\Sigma x, p_{n}(\delta)\right\rangle=0$. Since $H^{4 k-1}(X)$ is free for all $k \geqq 1$, this means that for all $n \geqq 1, p_{n}(\delta)=0$. Since $K O^{3}(X)$ is free, this implies $\delta=0$, that is, $\xi=0$. Therefore $\Phi(X)$ is monic. Since by Lemma $2.2 \Phi(X)$ is onto, this completes the proof of Theorem 1.

Proof of Theorem 2. The proof of (1) is easy. We shall prove (2). Note that $\left[\Sigma^{4 n} Q^{m}, S p\right] \cong\left[\Sigma^{4 n+1} Q^{m}, B S p\right] \cong K O^{3-4 n}\left(Q^{m}\right)$. Let $g^{\prime}(n, s): \Sigma^{4 n+1} Q^{m} \rightarrow B S p$ be the map which corresponds to $\alpha_{n+s-1} x_{s} \in K O^{3-4 n}\left(Q^{m}\right)$. Then the stable map $g(n, s)$ is represented by the following composite;

$$
\Sigma^{\infty} \Sigma^{4 n} Q^{m} \stackrel{\Sigma^{\infty}\left(\operatorname{adj} g^{\prime}(n, s)\right)}{\longrightarrow} \Sigma^{\infty} \Omega B S p=\Sigma^{\infty} S p \stackrel{\operatorname{adj} \theta}{\longrightarrow} \Sigma^{\infty} Q^{\infty} .
$$

From Theorem 1 and its proof, all we have to do is: 


$$
\begin{aligned}
& \left\langle g^{\prime}(n, s)_{*} r_{k}, p_{n+k}\right\rangle \\
& =\left\langle\gamma_{k}, g^{\prime}(n, s)^{*} p_{n+k}\right\rangle \\
& =\frac{(2 n+2 k) !}{2 n+2 k}\left\langle\gamma_{k}, p h\left(g^{\prime}(n, s)\right)\right\rangle \\
& =(2 n+2 k-1) !\left\langle r_{k}, p h\left(\alpha_{n+s-1} x_{s}\right)\right\rangle \\
& =a(n+s-1) \frac{(2 n+2 k-1) !}{(2 k-1) !}(2 s-1) ! M(k, s) \quad \text { (By Theorem 2.1 [7]). }
\end{aligned}
$$

Thus we have the desired result.

Similarly (3) and (4) are proved. This completes the proof of Theorem 2.

Proof of Theorem 4. We shall prove (1). Let $\xi \in K O^{4}(X)$ and $x \in H_{4 n}(X)$. We denote the Bott map: $\Sigma^{8} B S p \rightarrow B S p$ by $B$. Then it is enough to show that

$$
f_{*}(\Psi(X)(\xi)(x))=\Psi(B(\xi))\left(\Sigma^{8} x\right)
$$

L.H.S. $=f_{*}\left(\left\langle x, P^{\Delta_{n}}(\xi)\right\rangle \beta_{n}\right) \quad($ By Lemma 2.3)

$$
=\left\langle x, P^{\Delta_{n}}(\xi)\right\rangle f_{*}\left(\beta_{n}\right)
$$

$$
=\frac{(2 n+4) !}{(2 n) !}\left\langle x, P^{\Delta_{n}}(\xi)\right\rangle \beta_{n+2} \quad(\text { By Proposition 3). }
$$

R.H.S. $=\left\langle\Sigma^{8} x, P^{\Delta_{n+2}}(B(\xi))\right\rangle \beta_{n+2}$

$$
\begin{aligned}
& =\left\langle\Sigma^{8} x, \frac{(2 n+4) !}{2} p h(B(\xi))\right\rangle \beta_{n+\varepsilon} \\
& =\frac{(2 n+4) !}{2} \cdot \frac{2}{(2 n) !}\left\langle x, P^{\Delta_{n}}(\xi)\right\rangle \beta_{n+2} \\
& =\text { L.H.S. }
\end{aligned}
$$

The proofs of (2) and (3) are similar. We omit them.

\section{§3. Remarks and Problems}

As we cited, there exists a complex version of theorems in $\S 1$. In this section we shall give some remarks about the commutativity with the complex version. Let $c: K O^{*}(X) \rightarrow K^{*}(X)$ be the complexification map. Let $h: \mathbb{H} P^{\infty}$ $\rightarrow C P^{\infty}$ be the stable map which corresponds to $c(x) \in K^{4}\left(H P^{\infty}\right)$. Then;

\section{Proposition 3.1.}

$$
h_{*}\left(\beta_{k}\right)=2 \delta_{2 k}
$$

where $\delta_{i}$ is the standard generator of $H_{2 i}\left(C P^{\infty}\right)$. Moreover the following diagram 
commutes;

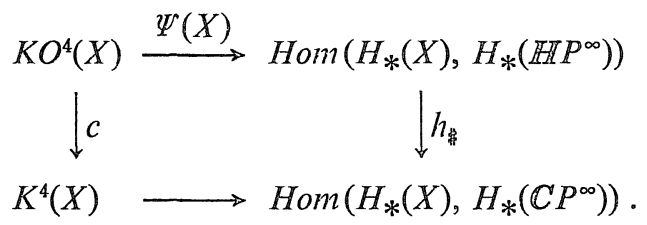

Proof of the proposition is easy. So we omit it. It is not clear that the map $h$ coincides to the Becker-Gottlieb transfer of the $S^{2}$ bundle $\mathbb{C} P^{\infty} \rightarrow \mathbb{H} P^{\infty}$.

Finally I would like to give rise to a problem;

In the complex case the corresponding map to the complex Bott map $\Sigma^{2} B U \rightarrow$ $B U$ can be realized as an unstable map $\Sigma^{3} \mathbb{C} P^{\infty} \rightarrow \Sigma \mathbb{C} P^{\infty}$. It seems plausible that the stable maps $f: \Sigma^{8} \mathbb{H} P^{\infty} \rightarrow \mathbb{H} P^{\infty}$ and $g: \Sigma^{8} Q^{\infty} \rightarrow Q^{\infty}$ can be realized as unstable maps $\Sigma^{9} \mathbb{H} P^{\infty} \rightarrow \Sigma \mathbb{H} P^{\infty}$ and $\Sigma^{8} Q^{\infty} \rightarrow Q^{\infty}$, respectively.

\section{References}

[1] Becker, J.C., Charactcristic classes and K-theory, Lecture Notes in Math., 428 (1978), 132-143.

[2] Crabb, M.C. and Knapp, K., On the codegree of negative multiples of Hopf bundles, Proc. Royal Soc. Edinburgh, 107A, (1987). 87-107.

[3] James, I.M., The topology of the Stiefel manifolds, London Math. Soc. Lecture Note Series, 24 (1976).

[4] McGibbon, C.A., Self maps of projective spaces, Trans. Amer. Math. Soc., 271 (1982), 326-346.

[5] Milnor, J. and Moore, J.C., On the structure of Hopf algebras, Ann. Math., 81 (1965), 221-264.

[6] Morisugi, K., Stable self maps of the quaternionic (quasi-) projective space, Publ. RIMS, Kyoto Univ., 20 (1984), 971-976.

[7] - Metastable homotopy groups of Sp(n), J. Math. Kyoto, Univ. 27 (1987), 367-380.

[8] Segal, G., On the stable homotopy of complex projective space, Quart. J. Math., Oxford (2), 24 (1973), 1-5.

[9] Walker, G., Estimates for the complex and quaternionic James numbers, Quart. J. Math., 32 (1981), 467-489. 
